\title{
Barreiras cognitivas: uma perspectiva decisiva para promover estratégias de conhecimento no desempenho da atividade organizacional
}

\author{
Cláudio Reis Gonçalo \\ Doutor em Engenharia de Produção - UNISINOS \\ cgoncalo@unisinos.br
}

Este trabalho propõe que estratégias de conhecimento sejam avaliadas a partir das barreiras cognitivas existentes nas organizações. Considera-se que a identificação dessas barreiras seja uma perspectiva decisiva para a tomada de decisão gerencial quanto à criação e transferência de conhecimento no desempenho da atividade. O reconhecimento desta perspectiva identifica fatores decisivos quanto a promover e/ou manter capacidades estratégicas da organização. Em um estudo de caso exploratório, em empresa envolvida com inovação tecnológica, a investigação das barreiras cognitivas permitiu reconhecer decisões estratégicas emergentes, principalmente, para "eliminar" as barreiras existentes relativas à armazenagem e distribuição do conhecimento. Na análise do estudo de caso, observou-se como fator crítico de sucesso a forma como os líderes valorizam o contexto e, em especial, como promovem o exercício da autonomia e de atividades informais para obterem estratégias de conhecimento eficazes.

Palavras-chave: barreiras cognitivas; estratégia de conhecimento; diagnóstico organizacional.

This article presents empirical research of an organizational perspective for the promotion of knowledge strategies. The organizational perspective is defined by the cognitive barriers to assist managerial decisions of knowledge strategies. Recognizing cognitive barriers facilitates the decisions in relation to the promotion or sustenance of strategic capabilities in the organization. This study was built in a longitudinal case study examining key success factors for better promotion of knowledge strategies. The investigated company, involved on technological innovation, could recognize its cognitive barriers and choose emergent strategic decisions in order to eliminate the existent cognitive barriers for creating and transfering knowledge. In the analysed case, it was observed success critical factors such as how leaders value the context and promote autonomy and informal activities for obtaining effective knowledge strategies.

Keywords: cognitive barriers; knowledge strategy; organizational diagnostic.

\section{Introdução}

Estratégias de conhecimento vêm sendo investigadas em um campo emergente de pesquisa, em particular para empresas cujo posicionamento estratégico está diretamente relacionado ao conhecimento como gerador de vantagem competitiva. A atribuição gerencial de promover estratégias de conhecimento assume um papel decisivo, principalmente para aquelas organizações nas quais a competitividade baseia-se em suas cadeias de conhecimentos.

Este trabalho apresenta uma perspectiva organizacional sob a ótica de barreiras cognitivas com o objetivo de contribuir para as tomadas de decisões gerenciais relativas à complexa função de criar e transferir conhecimento nas organizações. Reconhecer as barreiras existentes possibilita identificar as dificuldades para que o conhecimento flua" naturalmente na organização. Propõe-se que ao gerente caberá tomar decisões para eliminar ou reduzir essas barreiras cognitivas, assumindo o pressuposto básico que a sua principal atividade em relação à promoção de estratégias de conhecimento seja criar condições para que as pessoas acessem e desenvolvam o conhecimento individual e coletivo.

Estratégia e conhecimento vêm sendo estudados em um emergente campo de pesquisa reconhecido como uma visão da empresa baseada em conhecimento (NONAKA, 1995; SPENDER, 1996; GRANT, 1997; SVEIBY, 2001) que é uma combinação entre estudos da teoria de estratégia - baseada em recursos e capacidades - e de epistemologia. A teoria baseada em recursos e capacidades enfatiza o lado interno da organização como diferencial estratégico competitivo (BARNEY, 
1991; PRAHALAD e HAMEL, 1990; LEONARD-

BARTON, 1992), enquanto que a epistemologia suporta os pressupostos necessários para a prática e a pesquisa da gestão do conhecimento (VENZIN et al., 1998).

A análise das capacidades estratégicas é uma alternativa para compreender como as organizações se destacam com base na forma de fazer as coisas" e, conseqüentemente, estabelecer e/ou promover os conhecimentos requisitados (PRAHALAD e HAMEL, 1990; LEONARDBARTON, 1992; TEECE et al., 1997; MAKADOK, 2001). As capacidades estratégicas serão consistentes quando forem reconhecidas pelo cliente e, portanto, necessitam ser construídas através de um longo processo de evolução. O gerente tem um poder limitado para criar" novas capacidades. Propõe-se que uma alternativa seja promover" as capacidades estratégicas através do reconhecimento de suas barreiras cognitivas, e na conseqüente identificação de ações para reduzi-las ou eliminálas. Desta maneira, espera-se que este trabalho contribua tanto para facilitar tomadas de decisões que objetivem o melhor uso das capacidades estratégicas existentes, como para facilitar tomadas de decisões que vislumbrem novas capacidades estratégicas pretendidas.

Este trabalho apresenta uma proposta de análise organizacional sob a ótica de barreiras cognitivas para a promoção de estratégias de conhecimento. Para tanto, propõe-se a analisar os conceitos aplicados e a estrutura de pesquisa desenvolvida; os procedimentos metodológicos, e a identificação e análise das barreiras encontradas na empresa investigada; e, por fim, uma análise geral do estudo de caso.

\section{Barreiras cognitivas: as dificuldades para promover estratégias de conhecimento}

Estratégia, nesta pesquisa, significa um conjunto de ações com sentido específico, que se expressam na maneira peculiar de cada organização em fazer as coisas para atingir um sistema de objetivos, equivalente ao que Mintzberg (1987) chama de perspectiva ou o modo como as pessoas da organização vêem o mundo. Neste sentido, estratégia foi investigada como um conjunto coerente de ações individuais para viabilizar um sistema de objetivos, conjunto este que é suportado como um ativo por uma auto-sustentável massa crítica de opiniões na organização (EDEN e ACKERMANN, 1998). Este conceito de estratégia está relacionado, diretamente, com o conceito de cognição aplicado neste trabalho.

Investiga-se a cognição representada pelo cotidiano através das relações interpessoais e coordenações de ações, ou seja, através das revelações sobre o que se faz. e como se opera na atividade (MATURANA, 2001). Estudando a compreensão" dessas práticas pensantes, analisa-se a complexidade organizacional através de processos cognitivos (WEICK, 1995). Esses processos pertencem, conseqüentemente, aos sistemas representativos da inteligência organizacional. Em uma perspectiva cognitiva, considera-se que inteligência organizacional é o potencial da organização para criar e transferir conhecimentos gerando uma maneira peculiar de relacioná-los para encontrar soluções criativas e inovadoras nas tomadas de decisões (MARCH, 1999; WAGNER e STENBERG, 1986; PÓR, 1995; WIIG, 1993; LEONARDBARTON, 1992; QUINN et al.,1996; WEICK e ROBERTS, 1993).

Compreendem-se como barreiras cognitivas as barreiras existentes na organização para o uso da sua inteligência; ou seja, quanto menores forem as barreiras cognitivas maior será a possibilidade da aplicação da inteligência organizacional. O reconhecimento das barreiras cognitivas permitirá ao gerente tomar as decisões necessárias para reduzi-las ou eliminá-las, podendo assim promover estratégias eficazes de conhecimento.

Uma organização atua de forma inteligente quando, a partir de seu potencial (inteligência), consegue superar as suas barreiras cognitivas para encontrar soluções adequadas ao contexto do problema vivenciado. Para tanto, a estratégia de conhecimento necessária estará diretamente relacionada com o contexto de inteligência, e será definida e reconhecida em uma determinada perspectiva cognitiva da organização. Neste sentido, estratégia de conhecimento significa o conjunto de ações peculiares relacionadas com os processos de criação e transferência de conhecimento que ocorrem no contexto cognitivo do uso da inteligência organizacional.

A construção de uma capacidade estratégica ocorrerá a partir do exercício da atividade, desde que esta seja promovida para o desenvolvimento de habilidades cognitivas que diferenciem a organização. Considerando os conceitos discutidos e estudos de performance organizacional relacionados com estratégia, apresenta-se a seguir como pesquisar as barreiras cognitivas em uma organização.

\section{Como revelar as barreiras cognitivas nas organizações?}

Estudos sobre performance organizacional relacionados com estratégia vêm sendo realizados incluindo o contexto entre as dimensões de análise, além do conteúdo e o processo (PETTIGREW e WHIPP, 1994; KETCHEN et al., 1996; WIT e MEYER, 1998). A estrutura desta pesquisa baseia-se em examinar estas três dimensões 
essenciais, que para o foco em conhecimento organizacional (ver Figura 1) configuram-se como:

- o conteúdo - o conhecimento organizacional, que significa o conhecimento como o próprio produto (foco) da estratégia, elucidando-o em o que" representa em suas diversas categorias;

- o contexto - o contexto cognitivo na perspectiva da inteligência organizacional, que significa as variáveis do contexto interno que influenciam/ viabilizam o desenvolvimento do conhecimento através de processo estratégico proposto;

- o processo - o desenvolvimento do conhecimento, que significa o como" o conhecimento se desenvolve na organização.

\subsection{O conteúdo: o conhecimento conceitual}

Todas as perspectivas do conhecimento, consideradas no quadro da Figura 2 apresentado a seguir, dizem respeito ao nível cognitivo, ou seja, em cada perspectiva se propõe investigar o como", o que" e o porquê" acontece. Não se pretende analisar o conhecimento propriamente dito do produto ou serviço envolvidos e sim como se obtém conhecimento para desempenhar a atividade (PROCESSO), o que é esse conhecimento para a ativi-dade (CONTEÚDO) e por que o conhecimento é assim desenvolvido (CONTEXTO).

Na perspectiva sistêmica, o conhecimento está no próprio processo, por exemplo, as pessoas têm o conhecimento de trabalhar através de projetos, ou de compartilhar a informação por teleconferências, ou de trabalhar em rede, ou de construir equipamentos por encomenda. Na perspectiva capacidade, o conhecimento é o potencial para influenciar a ação; é aquele conhecimento gerador do potencial que diferencia a organização para a atuação em determinado tipo de processo. Na perspectiva prática profissional, é o conhecimento automático, aquele conhecimento que se tornou tácito pelo exercício do dia-a-dia do trabalho.

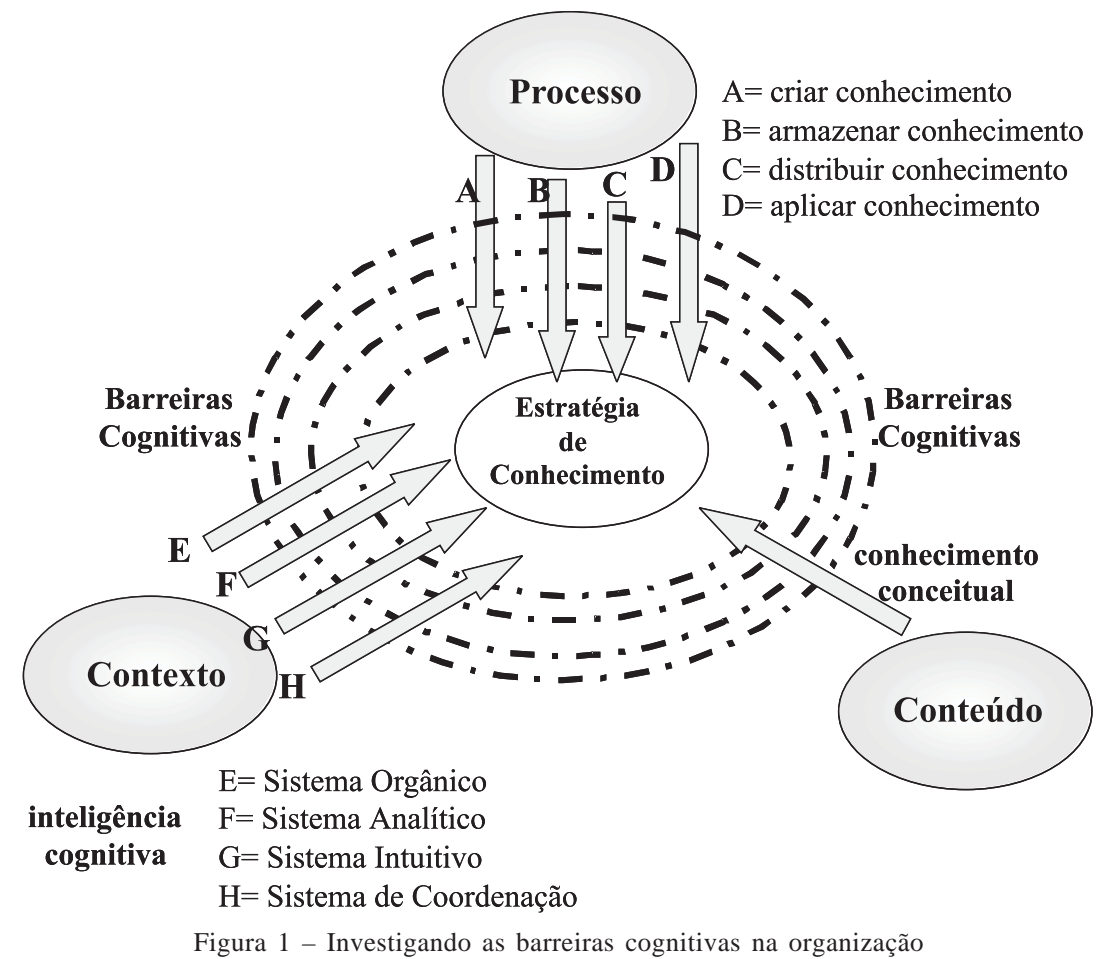

\begin{tabular}{|c|l|l|}
\hline Perspectivas & \multicolumn{1}{|c|}{ Significado do conhecimento } & Implicações para a gestão do conhecimento \\
\hline Sistêmica & $\begin{array}{l}\text { O conhecimento está na experiência do } \\
\text { processo de aplicação propriamente dito. }\end{array}$ & $\begin{array}{l}\text { O foco está no fluxo de conhecimento e no processo de } \\
\text { criar, armazenar, compartilhar e distribuir conhecimento. }\end{array}$ \\
\hline Capacidade & O conhecimento é o potencial para subsidiar a ação. & $\begin{array}{l}\text { O foco é a construção estratégica de competências } \\
\text { centrais e know-how na organização. }\end{array}$ \\
\hline Prática Profissional & O conhecimento está no desempenho da atividade. & $\begin{array}{l}\text { O foco é promover um ambiente de aprendizagem } \\
\text { individual/organizacional. }\end{array}$ \\
\hline
\end{tabular}

Fonte: Baseado em Alavi (2001), Wigg (1993) e Zack (1999).

Figura 2 - O conteúdo: o conhecimento conceitual 


\subsection{O processo: a implementação estratégica}

Analisa-se estratégia de conhecimento em duas dimensões: a implementação estratégica e a formulação estratégica (ver Figura 3). Propõe-se que a prioridade desta estrutura de pesquisa baseie-se no nível tático, ou seja, na própria implementação estratégica (GLADSTEIN e QUINN, 1985), que através do processo de gestão do conhecimento (ALAVI, 2001) promova ações para criar e transferir conhecimento no desempenho das atividades (NONAKA et al., 2001). Este processo engloba as seguintes etapas:

a) adquirir/criar conhecimento - engloba tanto as fontes do conhecimento como as condições facilitadoras para a atuação das pessoas, tanto a nível individual como organizacional;

b) explicitar/armazenar conhecimento - envolve captar o conhecimento explícito das pessoas da organização, codificá-lo e armazená-lo para o possível acesso posterior;

c) aplicar - caracteriza o uso do conhecimento para melhoria da performance organizacional, através do seu efetivo emprego na atividade e a possibilidade de aprender-fazendo";

d) distribuir conhecimento - envolve a transferência da parte explícita do conhecimento das pessoas, utilizando ou não estratégias técnicas para facilitar o seu acesso por todos na organização.

Quanto à dimensão de formulação estratégica, esta significa a difícil etapa de definição do conhecimento que a organização necessitará no futuro, ou seja, os requisitos de conhecimento para atender a novas decisões estratégicas. A decisão de adquirir um novo conhecimento somente pode ser exequíivel a partir da decisão de promover as condições para as pessoas pesquisarem na direção do conhecimento pretendido. Portanto, a decisão de formulação estratégica envolve os requisitos para a promoção das capacidades organizacionais. A formulação estratégica preocupa-se em promover as condições para minimizar o gap cognitivo da organização, a partir da alavancagem" dos recursos necessários para promover a capacidade desejada (QUINN, 1999), e de constantes ações gerenciais para redução das barreiras cognitivas.

\subsection{O contexto: a expressão da inteligência organizacional}

O contexto envolve os sistemas cognitivos representativos da expressão da inteligência organizacional (GONÇALO et al., 2002). Sob a perspectiva cognitiva, os sistemas podem ser classificados em: orgânico, analítico, intuitivo e de coordenação. O Sistema Orgânico representa a atuação da organização frente ao profissional como elemento individual e social (GRATTON, 2000). O Sistema Analítico identifica as condições e capacidades existentes para desenvolver o conhecimento estruturado.

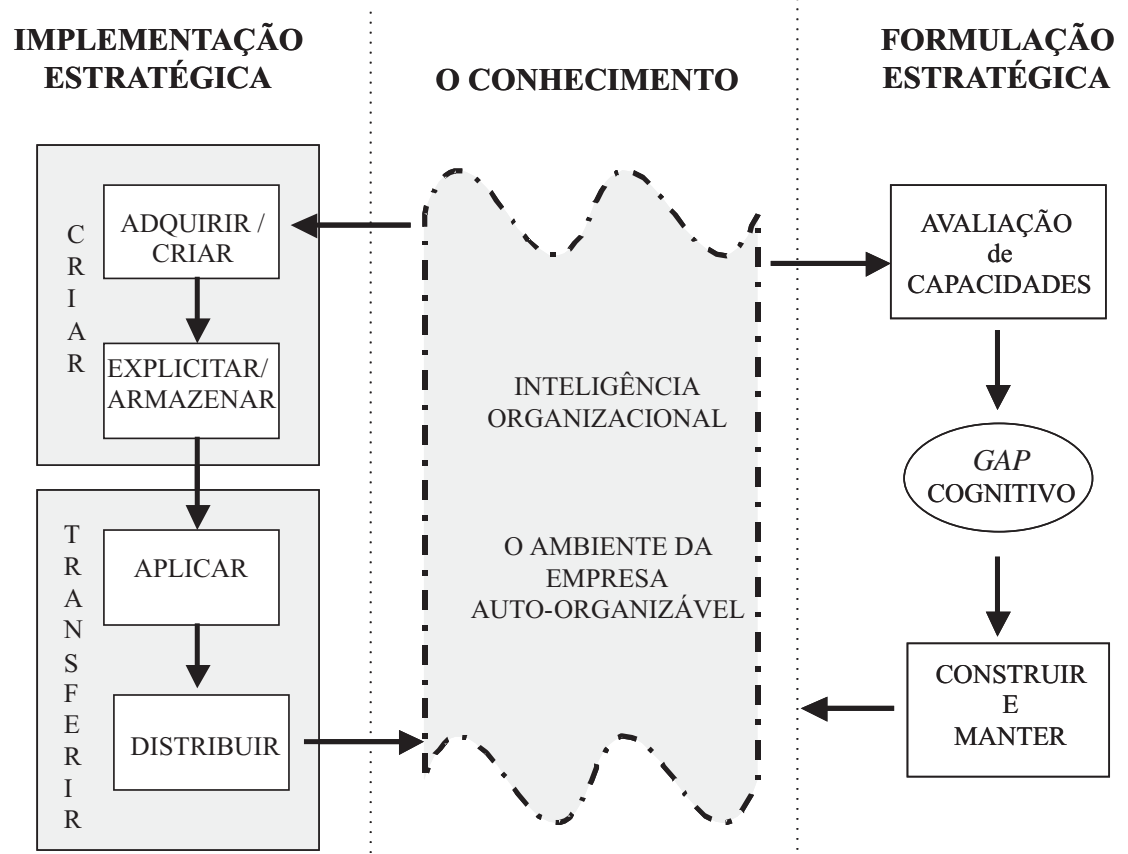

Fonte: Baseado em Alavi(1997), Buckowitz e Williams (2000), Von Krogh et al. (2000), Nonaka et al. (2001)

Figura 3 - O processo de estratégia de conhecimento organizacional 
O Sistema Intuitivo retrata o tratamento dedicado ao conhecimento tácito com base na experiência e intuição dos indivíduos, e o Sistema de Coordenação engloba as características da liderança organizacional com objetivo de interligar e promover entre as pessoas uma rede natural de conhecimentos.

Cada sistema de inteligência foi dividido em dimensões essenciais, que significam as ênfases assumidas como contexto para a pesquisa. Para cada dimensão focalizouse uma barreira potencial considerada como crítica para a promoção de cada sistema de inteligência, através de estratégia de conhecimento (ver quadro 2 da Figura 4 a seguir).

\section{A matriz das barreiras cognitivas}

A Matriz das Barreiras Cognitivas, ( Figura 5) identifica as barreiras resultantes da relação entre o processo de implementação estratégica do conhecimento (processo/ colunas) e os sistemas representativos da inteligência organizacional (contexto/linhas). Em relação à estrutura

\begin{tabular}{|c|c|c|}
\hline $\begin{array}{l}\text { Sistemas de } \\
\text { Inteligência }\end{array}$ & Dimensões Essenciais & $\begin{array}{l}\text { Barreiras Cognitivas Potenciais para a } \\
\text { promoção de estratégias de conhecimento }\end{array}$ \\
\hline \multirow{3}{*}{$\begin{array}{l}\text { SISTEMA } \\
\text { ORGÂNICO }\end{array}$} & Pessoas operam no tempo. & - falta de habilidades de criar estratégias. \\
\hline & Pessoas procuram por significados. & • falta de habilidades de pensamento sistêmico. \\
\hline & Pessoas precisam confiar. & - falta de habilidades de criar/assumir desafios. \\
\hline \multirow{3}{*}{$\begin{array}{l}\text { SISTEMA } \\
\text { ANALÍTICO }\end{array}$} & Conhecimento explícito & $\begin{array}{l}\text { - falta de habilidades em estimular o acesso e informação ao } \\
\text { conhecimento estruturado. }\end{array}$ \\
\hline & Memória organizacional & $\begin{array}{l}\text { - falta de habilidades na expressão da experiência individual na } \\
\text { construção da memória organizacional aplicando ou não a tecnologia } \\
\text { da informação. }\end{array}$ \\
\hline & Rede de informação/conhecimento & $\begin{array}{l}\text { - falta de habilidades de iniciativa em gerenciamento autônomo e de } \\
\text { compartilhar conhecimento. }\end{array}$ \\
\hline \multirow{2}{*}{$\begin{array}{l}\text { SISTEMA } \\
\text { INTUITIVO }\end{array}$} & $\begin{array}{l}\text { Conhecimento tácito/intuitivo } \\
\text { heurístico. }\end{array}$ & $\begin{array}{l}\text { - falta de habilidades em estimular o conhecimento não estruturado, } \\
\text { ou seja, baseado nas experiências das pessoas. }\end{array}$ \\
\hline & Inteligência na absorção da informação. & - falta de habilidades na escolha da absorção de melhores dados/informações. \\
\hline \multirow{2}{*}{$\begin{array}{l}\text { SISTEMA DE } \\
\text { COORDEN. }\end{array}$} & $\begin{array}{l}\text { Visão sistêmica de conhecimento } \\
\text { para o negócio. }\end{array}$ & $\begin{array}{l}\text { - falta de habilidades na percepção da cadeia de processos e } \\
\text { conhecimento. }\end{array}$ \\
\hline & Característica da liderança "consciente". & $\begin{array}{l}\text { - falta de habilidades de estímulo à "conversa organizacional" e } \\
\text { influência interpessoal. }\end{array}$ \\
\hline
\end{tabular}

Fonte: Gonçalo(2004, p.122)

Figura 4 - O contexto: a inteligência organizacional na perspectiva cognitiva

\begin{tabular}{|l|c|c|c|c|}
\hline Contexto & $\begin{array}{c}\text { Criar } \\
\text { Conhecimento }\end{array}$ & $\begin{array}{c}\text { Armazenar } \\
\text { Conhecimento }\end{array}$ & $\begin{array}{c}\text { Distribuir } \\
\text { Conhecimento }\end{array}$ & $\begin{array}{c}\text { Aplicar } \\
\text { Conhecimento }\end{array}$ \\
\hline Sistema Orgânico & $\mathrm{B}_{\mathrm{O} \times \mathrm{Cri}}$ & $\mathrm{B}_{\mathrm{O} \times \mathrm{Arm}}$ & $\mathrm{B}_{\mathrm{O} \times \mathrm{Dis}}$ & $\mathrm{B}_{\mathrm{O} \times \mathrm{Apl}}$ \\
\hline Sistema Analítico & $\mathrm{B}_{\mathrm{A} \times \mathrm{Cri}}$ & $\mathrm{B}_{\mathrm{A} \times \mathrm{Arm}}$ & $\mathrm{B}_{\mathrm{A} \times \mathrm{Dis}}$ & $\mathrm{B}_{\mathrm{A} \times \mathrm{Apl}}$ \\
\hline Sistema Intuitivo & $\mathrm{B}_{1 \times \mathrm{Cri}}$ & $\mathrm{B}_{1 \times \mathrm{Arm}}$ & $\mathrm{B}_{1 \times \text { Dis }}$ & $\mathrm{B}_{1 \times \mathrm{Apl}}$ \\
\hline Sistema de Coordenação & $\mathrm{B}_{\mathrm{C} \times \mathrm{Cri}}$ & $\mathrm{B}_{\mathrm{C} \times \mathrm{Arm}}$ & $\mathrm{B}_{\mathrm{C} \times \mathrm{Dis}}$ & $\mathrm{B}_{\mathrm{C} \times \mathrm{Apl}}$ \\
\hline
\end{tabular}

Figura 5 - Matriz das Barreiras Cognitivas

de pesquisa, subentende-se que o conteúdo atue como uma referência básica, sendo investigado em todas as barreiras encontradas.

Onde os elementos da matriz significam:

\section{Barreiras Cognitivas Orgânicas}

$\mathrm{B}_{\mathrm{O} \text { x Cri }}=$ Barreira cognitiva orgânica em criar conhecimentos

$\mathrm{B}_{\mathrm{O} \text { x Arm }}=$ Barreira cognitiva orgânica em armazenar conhecimentos

$\mathrm{B}_{\mathrm{O} \times \mathrm{Dis}}=$ Barreira cognitiva orgânica em distribuir conhecimentos

$\mathrm{B}_{\mathrm{OxApl}}=$ Barreira cognitiva orgânica em aplicar conhecimentos 


\section{Barreiras Cognitivas Analíticas}

$\mathrm{B}_{\mathrm{AxCri}}=$ Barreira cognitiva analítica em criar conhecimentos

$\mathrm{B}_{\mathrm{Ax} \mathrm{Arm}}=$ Barreira cognitiva analítica em armazenar conhecimentos

$\mathrm{B}_{\mathrm{A} \times \mathrm{Dis}}=$ Barreira cognitiva analítica em distribuir conhecimentos

$\mathrm{B}_{\mathrm{AxApl}}=$ Barreira cognitiva analítica em aplicar conhecimentos

\section{Barreiras Cognitivas Intuitivas \\ $\mathrm{B}_{\mathrm{I} \times \mathrm{Cri}}=$ Barreira cognitiva intuitiva em criar conhecimentos \\ $\mathrm{B}_{\mathrm{I} \mathrm{x} \mathrm{Arm}}=$ Barreira cognitiva intuitiva em armazenar conhecimentos \\ $\mathrm{B}_{\mathrm{IxD} \text { Dis }}=$ Barreira cognitiva intuitiva em distribuir conhecimentos \\ $\mathrm{B}_{\mathrm{IxApl}}=$ Barreira cognitiva intuitiva em aplicar conhecimentos}

\section{Barreiras Cognitivas de Coordenação}

$\mathrm{B}_{\mathrm{C} \times \mathrm{Cri}}=$ Barreira cognitiva de coordenação em criar conhecimentos

$\mathrm{B}_{\mathrm{CxArm}}=$ Barreira cognitiva de coordenação em armazenar conhecimentos

$\mathrm{B}_{\mathrm{C} \times \mathrm{Dis}}=$ Barreira cognitiva de coordenação em distribuir conhecimentos

$\mathrm{B}_{\mathrm{CxApl}}=$ Barreira cognitiva de coordenação em aplicar conhecimentos

Portanto, os elementos da Matriz representam as barreiras cognitivas existentes ao processo de implementação estratégica do conhecimento conceitual. A Matriz indica que, por exemplo, para criar conhecimento, a organização pode possuir barreiras cognitivas relacionadas ao sistema orgânico, ao sistema analítico, ao sistema intuitivo ou ao sistema de coordenação.

Cada elemento da Matriz, por possuir mais de uma categoria, representará um subconjunto. Por exemplo, as barreiras cognitivas analíticas em armazenar conhecimentos serão expressas por três categorias de análise: as habilidades relativas ao acesso e informação ao conhecimento estruturado (explícito); as habilidades na construção da memória organizacional e as habilidades de gerenciamento autônomo para a formação de redes de conhecimento. Portanto, $B_{A x A r m}$ será composta por três categorias de análise que auxiliam a identificação das barreiras analíticas para armazenar conhecimento na organização. Pretende-se que a construção da matriz, com base nas etapas exploratórias do estudo de caso, facilite a melhor compreensão de sua elaboração.

\section{Procedimentos metodológicos}

Adotou-se a pesquisa qualitativa para compreender uma situação específica na organização sob a ótica de existência de barreiras cognitivas, utilizando o contexto e o processo como dimensões básicas para a representação analisada. Este trabalho de pesquisa seguiu a linha filosófica interpretativa (MYERS, 2002). A pesquisa interpretativa considera que o acesso à realidade ocorre somente através de construções sociais tais como a linguagem, a consciência e o significados compartilhados. O objetivo desta pesquisa foi a interpretação dos significados organizacionais, daquilo que as pessoas percebem em relação às práticas aplicadas para criar e transferir conhecimentos.
Através da metodologia de estudo de caso, principalmente por ser adequado para a investigação de assuntos empíricos específicos, com uma estratégia de pesquisa abrangente, buscou-se:

a) investigar o contexto da organização para a promoção de conhecimento, na procura de compreender este contexto para" ou no" desempenho da atividade;

b) investigar barreiras cognitivas através de estratégias abrangentes de conhecimento, as quais requerem saber administrar muitas categorias de análise com um grande número de fontes de evidência.

O estudo de caso foi conduzido através de estratégia exploratória (YIN, 2001), na medida em que visou investigar um novo conceito de análise das organizações sob a perspectiva das barreiras cognitivas. O projeto total da pesquisa foi dividido em três etapas exploratórias: numa primeira etapa investigou-se o contexto, numa segunda, o processo e, por último, numa terceira etapa, o processo versus o contexto. A estratégia de pesquisa do projeto completo envolveu duas empresas (ALFA e BETA) cujo produtos finais dependem de inovação tecnológica. A empresa BETA não foi apresentada neste trabalho pois o objetivo foi de representar as barreiras cognitivas totais, enquanto que a sua investigação auxiliou para compreender uma etapa intermediária de representação do processo. Neste artigo, a partir da primeira e terceira etapas exploratórias, apresenta-se o estudo de caso da empresa ALFA.

\subsection{A coleta de evidências}

Para a investigação profunda da organização, combinouse diferentes técnicas de coleta de evidências: documentos/relatórios, entrevistas e observação direta. Como a pesquisa estava relacionada com a construção de teoria 
a partir de estudo de caso, foi freqüente a superposição entre a análise e a coleta de dados, conforme observa Eisenhardt (1989).

Diversos documentos foram utilizados para a coleta de evidências como a participação em prêmios, internet, reportagens, projetos desenvolvidos e, em especial, a análise de duas dissertações de mestrado que foram pesquisas recentes na organização.

As entrevistas foram do tipo semi-estruturada, auxiliadas pelo protocolo de pesquisa previamente elaborado. Procurou-se valorizar, intensamente, o momento de esclarecer o objetivo e abrangência da pesquisa ao entrevistado, de forma a proporcionar o contexto mais espontâneo possível para a sua expressão, e incentivar o relato de exemplos no exercício de sua atividade. Nas duas etapas exploratórias A e C, realizaram-se 28 entrevistas com todas as lideranças da organização, totalizando $38 \mathrm{~h}$ e $45 \mathrm{~min}$ de duração. Os líderes possuem formação superior, na sua grande maioria, ou estão em processo de obtê-la.

Quanto à observação, foi possível acompanhar as pessoas no desempenho de suas atividades em diferentes momentos, tais como sob pressão" de entrega do equipamento, ou mesmo na análise e reflexão sob uma possível nova contratação. Além disso, por solicitação da empresa após o término das entrevistas, o pesquisador coordenou e atuou como um dos professores em um projeto de desenvolvimento gerencial com 88 horasaula, com 25 participantes representando quase $90 \%$ da liderança. Esta atividade contribuiu para estender a observação como participante da construção de novos conhecimentos, assim como melhor compreender a dinâmica do comportamento da liderança frente ao novo conhecimento, neste contexto de aprendizagem.

\section{0 estudo de caso}

A empresa ALFA atua no setor de desenvolvimento, projeto e construção de linhas e sistemas de montagem e testes para fabricantes de autopeças, eletrodomésticos e componentes eletrônicos. Cada linha de montagem representa um novo projeto e a construção de um novo produto, específico para as necessidades dos clientes. Os produtos e serviços incluem soluções em montagem, teste e manuseio de peças, variando de uma bancada de teste a uma linha de montagem completa, totalmente automatizada. Fundada na década de 1980, a empresa possui hoje duas sedes, uma no Brasil e outra no exterior, e atualmente concentra-se em três estratégias consideradas fundamentais: focalização, confiabilidade e rapidez. A empresa diferencia-se pela sua capacidade de encontrar soluções tecnológicas inovadoras e criativas para os diversos problemas no seu foco de negócio.

A principal pergunta de pesquisa que orientou este estudo de caso foi: como identificar as barreiras cognitivas frente ao uso da inteligência organizacional através de estratégias de conhecimento?

Para desenvolver a investigação, duas etapas exploratórias foram realizadas:

- na primeira, pesquisou-se como a organização pode ser analisada segundo o contexto cognitivo para promover estratégias de conhecimento (conforme item 3.2);

- na segunda, como estratégias de conhecimento ocorrem ou se expressam no desempenho das atividades organizacionais (conforme item 3.3).

A partir da associação entre as evidências das duas etapas exploratórias construiu-se a Matriz das Barreiras Cognitivas.

\subsection{A matriz das barreiras cognitivas}

Observa-se que o processo de construção das Matrizes ocorreu de forma interativa entre as categorias pesquisadas a partir das etapas exploratórias. Para simplificar a análise, apresenta-se, a seguir, as Matrizes das Barreiras Cognitivas (Figura 6), explodindo" e apreciando os seus principais elementos com base nas etapas exploratórias realizadas.

\begin{tabular}{|c|c|c|c|c|c|}
\hline \multicolumn{2}{|l|}{ Contexto } & Criar & Armazenar & Distribuir & Aplicar \\
\hline \multirow{3}{*}{$\begin{array}{l}\text { SISTEMA } \\
\text { ORGÂNICO }\end{array}$} & $\begin{array}{l}\text { Habilidades de criar } \\
\text { estratégias - } 01\end{array}$ & $\square$ & $\square$ & $\square$ & $\square$ \\
\hline & $\begin{array}{l}\text { Habilidades de pensamento } \\
\text { sistêmico - } \mathrm{O} 2\end{array}$ & $\operatorname{ma}$ & 口 & $\bullet$ & 口 \\
\hline & $\begin{array}{l}\text { Habilidades de criar/ } \\
\text { assumir desafios - O3 }\end{array}$ & $\square$ & $\square$ & $\square$ & $\square$ \\
\hline
\end{tabular}

= fraca barreira cognitiva

= média barreira cognitiva

= forte barreira cognitiva 
Observações sobre as fortes ou médias barreiras cognitivas:

1. Forte $\mathbf{B}_{\mathrm{O} 2 \times \text { Criar conhecimento }}$ caracterizada, em particular, pela dificuldade de participação de um maior número de pessoas na concepção de novos projetos.

2. Média $\mathbf{B}_{\mathrm{O} 2 \times \text { Distribuir conhecimento }}$ caracterizada tanto pela velocidade de atuação na atividade e/ou pela alta especialidade funcional de algumas atividades.

\begin{tabular}{|c|c|c|c|c|c|}
\hline \multicolumn{2}{|c|}{ Contexto Processo } & Criar & Armazenar & Distribuir & Aplicar \\
\hline \multirow{3}{*}{$\begin{array}{l}\text { SISTEMA } \\
\text { ANALÍTICO }\end{array}$} & $\begin{array}{l}\text { Habilidades em estimular } \\
\text { o acesso e informação ao } \\
\text { conhecimento estruturado } \\
\text { - A1 }\end{array}$ & $\square$ & $\bullet \bullet$ & 口 & $\square$ \\
\hline & $\begin{array}{l}\text { Habilidades na expressão } \\
\text { da experiência individual } \\
\text { na construção da memória } \\
\text { organizacional - A2 }\end{array}$ & $\square$ & m & $\square$ & -1 \\
\hline & $\begin{array}{l}\text { Habilidades de iniciativa } \\
\text { em gerenciam. autônomo } \\
\text { e de compartilhar } \\
\text { informação - A3 }\end{array}$ & 口 & $\bullet$ & $\square$ & $\square$ \\
\hline
\end{tabular}

Figura 6. b) Matriz das Barreiras Cognitivas Analíticas

Observações sobre as fortes ou médias barreiras cognitivas:

1. Média $\mathbf{B}_{\mathrm{A} 1 \text { x Armazenar conhecimento }}$ caracterizada, por exemplo, pela falta de um sistema de informação que auxilie o acesso a soluções de problemas resolvidos anteriormente e/ou características de projetos inovadores;

2. Forte $\mathbf{B}_{\mathrm{A} 2 \text { x Armazenar conhecimento }}$ caracterizada, por exemplo, por poucas iniciativas dos líderes em promover situações para relatos, descrição de experiências e organização dessas informações, justificado por eles pela falta de tempo devido à pressão" em obter resultados;

3. Média $\mathbf{B}_{\mathrm{A} 3 \times \text { Armazenar conhecimento }}$ caracterizada, por exemplo, por pouca padronização de procedimentos ou de processos de trabalho que dificultam a atuação para armazenar conhecimento/informação e facilitar o seu compartilhamento. Um exemplo identificado pela liderança é a falta de um modelo de gerenciamento de projetos que reduz a capacidade de armazenar conhecimentos/informações específicas cujos conceitos poderiam ser utilizados em outras situações.

4. Forte $\mathbf{B}_{\mathrm{A} 2 \times \text { Aplicar conhecimento }}$ caracterizada, por exemplo, pelos poucos esforços estruturados em analisar e/ou documentar procedimentos de sucessos e de fracassos na execução após cada construção de uma linha de produção (identificados como fechamentos de máquinas) e/ou na elaboração desses projetos.

\begin{tabular}{|c|c|c|c|c|c|}
\hline \multicolumn{2}{|l|}{ Contexto } & Criar & Armazenar & Distribuir & Aplicar \\
\hline \multirow{2}{*}{$\begin{array}{l}\text { SISTEMA } \\
\text { INTUITIVO }\end{array}$} & $\begin{array}{l}\text { Habilidade em estimular } \\
\text { o conhec. não estrut., } \\
\text { baseando-se na experiência } \\
\text { das pessoas - I1 }\end{array}$ & $\square$ & $\square$ & $\square$ & $\square$ \\
\hline & $\begin{array}{l}\text { Habilidades na escolha } \\
\text { da absorção de melhores } \\
\text { dados e/ou informações - } 12\end{array}$ & $\square$ & $\square$ & $\bullet$ & $\square$ \\
\hline
\end{tabular}

Figura 6. c) Matriz das Barreiras Cognitivas Intuitivas 
Observações sobre a média barreira cognitiva:

1. Média $\mathbf{B}_{\mathrm{I} 2 \times \text { Distribuir conhecimento }}$ caracteriza-se, por exemplo, pelos reduzidos momentos estruturados para compartilhar conhecimentos, como o mencionado seminário da engenharia" que permitiria o exercício da diversidade de informações para desenvolver a intuição heurística.

\begin{tabular}{|c|c|c|c|c|c|}
\hline \multicolumn{2}{|c|}{ Contexto Processo } & Criar & Armazenar & Distribuir & Aplicar \\
\hline \multirow{2}{*}{$\begin{array}{l}\text { SISTEMA DE } \\
\text { COORDEN. }\end{array}$} & $\begin{array}{l}\text { Habilidades na percepção } \\
\text { da cadeia de processos e } \\
\text { conhecimento - C1 }\end{array}$ & 口 & "I. & $\bullet$ & $\bullet$ \\
\hline & $\begin{array}{l}\text { Habilidades de estímulo à } \\
\text { conversa organizacional e } \\
\text { influência interpessoal - C2 }\end{array}$ & 口 & 口 & $\bullet \bullet$ & 口 \\
\hline
\end{tabular}

$=$ fraca barreira cognitiva

= média barreira cognitiva

$=$ forte barreira cognitiva

Figura 6. d) Matriz das Barreiras Cognitivas de Coordenação

Observações sobre as fortes ou médias barreiras cognitivas:

1. Forte $\mathbf{B}_{\mathrm{Cl} \text { x Armazenar conhecimento }}$ caracterizada, por exemplo, pela inexistência de um modelo de gestão de projetos aplicado por todos o qual facilitaria a armazenagem de informações com uma referência para comparação de resultados nas atividades pertencentes ao processo gerencial. Outras caracterizações desta barreira cognitiva são, por exemplo, a pouca memória estruturada de propostas (cotações) de linhas de produção, ou mesmo as poucas oportunidades planejadas para troca de experiências entre as fases de concepção, programação, projeto e execução da linha de produção.

2. Média $\mathbf{B}_{\mathrm{C} 1 \text { x Distribuir conhecimento }}$ caracterizada, por exemplo, pela alta velocidade de atuação da liderança e da equipe. Impulsionada por ser a rapidez de atuação um foco estratégico, as pessoas possuem pouco tempo para perceberem todos os processos e os conhecimentos associados.

3. Média $\mathbf{B}_{\mathrm{C} 2 \times \text { Distribuir conhecimento }}$ caracterizada, por exemplo, pela falta de um sistema de informações que auxilie a necessidade de discussões informais, gerando estruturas de apoio ao conhecimento compartilhado. Uma maneira seria a existência de um sistema de indicadores disponibilizados na Intranet que criaria tanto a discussão e aprendizagem informal como momentos formais de análise das causas do desempenho ocorrido na atividade e/ou no projeto.

4. Média $\mathbf{B}_{\mathrm{C} 1 \text { x Aplicar conhecimento }}$ caracterizada, por exemplo, pela necessidade de um sistema de gestão de projetos e/ou um sistema de controle de gestão (indicadores), ambos já identificados pela diretoria.

\subsection{Análise geral do estudo de caso}

O processo de condução do estudo de caso, com duas etapas exploratórias de investigação e períodos longitudinais de observação e análise, é que permitiu identificar as barreiras cognitivas apresentadas no item 6.1. O nível de intensidade das barreiras cognitivas encontradas foi avaliado de forma qualitativa. Para cada categoria foram utilizadas fontes múltiplas de evidências e, também, o encadeamento dessas evidências entre as diferentes etapas do processo investigado. No item 6.1, para barreiras cognitivas com nível médio e forte foram identificadas algumas dessas principais evidências.
As Matrizes das Barreiras Cognitivas possibilitaram identificar diferentes tipos de obstáculos existentes na organização ALFA. Com esta radiografia”, os gerentes dessa organização expressaram, no primeiro momento, algumas surpresas, mas, por outro lado, quase que imediatamente identificaram elementos críticos de mudança estratégica.

Entre os elementos decisivos representados nas Matrizes, ressaltam-se as fortes barreiras existentes em armazenar e aplicar o conhecimento frente às habilidades na construção da memória organizacional. Isto provocou a decisão dos Diretores da Empresa em intensificar ações para 
criar sistemas de padronização e/ou descrição dos conhecimento adquiridos, além da detalhada especificação dos projetos que naturalmente é realizada.

[...] a fonte muito grande de problemas que a gente tem hoje é essa, quer dizer, muda-se a especificação do projeto, parte do projeto, alguma característica profissional, e algum profissional dessas áreas: projeto mecânico, elétrico, projeto de software, projeto pneumático, não tem acesso a essa informação. Não fica sabendo, então, quando passa a se executar esse projeto as interfaces entre os diversos projetos não fecham, não batem, e surge o problema pra ser resolvido, gera ineficiência, gera atraso, gera custos [...]. (Gerente A)

Outro elemento considerado crítico foi a forte barreira cognitiva para criar conhecimentos em função das habilidades de pensamento sistêmico. A alta pressão" por atender a opção estratégica de rapidez leva cada grupo responsável por determinado projeto a focalizar seus esforços na gestão da implantação. Quanto a novas demandas de clientes que exijam diferentes alternativas de soluções, a participação acaba sendo restrita ao grupo de análise de diferentes aplicações.

[...] então, a engenharia de aplicações dá uma boa formatada no projeto. Ela já determina uma série de soluções por experiências anteriores acumuladas pela empresa. Quando não existe essa experiência, né? Nós somos consultados [...] Então, de fato nessa etapa nós não participamos, e é uma etapa fundamental. Na verdade, existe assim, uma certa [...] é uma área de atrito, é uma área de conflito. O pessoal questiona um pouco mais porque faria de uma forma diferente, porque enxergou uma solução um pouco diferente e aí existem limitações de custo e nem sempre é possível usar uma solução mais avançada tecnologicamente [...]. (Gerente B)

Se por um lado reconhecer as barreiras cognitivas médias e fortes estabelece uma percepção de problemas, por outro, reconhecer a existência de fracas barreiras enfatiza elementos positivos de gestão para a promoção de conhecimento na organização. No estudo de caso apareceram muitos desses elementos positivos, que auxiliam na explicação do sucesso que a empresa vem obtendo na produção de produtos inovadores. Entre 40 elementos investigados (número total de elementos das matrizes), 29 apresentaram fracas barreiras cognitivas, senão, não percebíveis.

Entre as fracas barreiras cognitivas, ou seja, elementos que são considerados como positivos, ressaltam-se as percebidas na distribuição de conhecimento frente às habilidades de iniciativa em gerenciamento autônomo e de compartilhar informação. A empresa caracteriza-se por uma alta capacidade estratégica para inovação, baseada no exercício da autonomia na tomada de decisão e do comportamento proativo de seus profissionais.
[...] Eu diria assim - é uma das coisas básicas na empresa: não tem ninguém - não vais encontrar ninguém aqui - de má vontade de sentar e conversar e trocar idéias. [....] Outras pessoas que vieram de empresas muito grandes, eles comentam -interessante é isso: que todos têm essa predisposição pra parar o que tá fazendo na hora e te dar uma atenção. Tentar resolver o problema contigo, não achar que o seu problema é mais sério e que não pode dar uma atençãozinha pro lado [...]. (Gerente C)

Há a percepção da liderança quanto à disposição entre as pessoas para compartilhar o conhecimento de que quanto mais se compartilha, mais respeito se obtém na equipe. Acreditam que a atividade desenvolvida na empresa promove o compartilhar, já que todos estão no mesmo barco" para produzir um produto sob encomenda, muitas vezes inédito em concepção e/ou tecnologia aplicada.

Também é necessário ressaltar que observou-se um alto nível de confiança na organização, o qual intensifica o relacionamento informal para a solução de problemas. Na Matriz das Barreiras Cognitivas observa-se as fracas barreiras relativas ao processo frente às habilidades de estimular o conhecimento não estruturado, baseando-se nas experiências das pessoas.

Se há uma palavra que represente o contexto da empresa, voltado para incentivar o acesso à informação e o comprometimento em resolver problemas, esta palavra é desafio. As pessoas assumem desafios na empresa investigada porque têm autonomia, podem errar, são valorizadas e confiam na organização. O contexto informal voltado para resolver problemas é percebido pelos clientes como uma capacidade estratégica, um diferencial muitas vezes reconhecido por todos que lhes agrega um alto valor competitivo na ação empreendedora para a inovação.

\section{Considerações finais}

Esta pesquisa desenvolveu uma perspectiva organizacional sob a ótica das suas barreiras cognitivas com o objetivo de identificar potenciais ou alternativas de gestão à promoção do conhecimento nas organizações. O estudo de caso apresentado auxiliou na construção teórica proposta, sendo que este trabalho focalizou os resultados da pesquisa que foram demonstrados através da Matriz das Barreiras Cognitivas como um instrumento de análise.

Não foi objeto deste trabalho detalhar a construção deste instrumento, e observa-se que há necessidade de desenvolver um maior número de estudos para a definição de parâmetros de avaliação dos níveis de complexidade (ou intensidade) de barreiras cognitivas. Constatou-se que a sua aplicação na empresa ALFA, mesmo demandando um profundo estudo organizacional, 
mostrou-se muito eficaz para a identificação das barreiras críticas para a promoção de estratégias de conhecimento, tais como: habilidades em pensamento sistêmico para criar conhecimentos, habilidades na construção da memória organizacional para armazenar e aplicar conhecimentos, e habilidades na percepção da cadeia de processos para armazenar conhecimentos.

Por outro lado, observou-se na empresa analisada que muitas barreiras foram consideradas como fracas, permitindo, também, inferir que estes elementos sejam representativos dos fatores de sucesso em estratégias de conhecimento, até então alcançadas. Reconhecê-los cria uma grande responsabilidade gerencial em relação à definição de alternativas para manter e/ou ainda melhor desenvolver esses diferenciais na organização.

Através da análise das Matrizes das Barreiras Cognitivas procurou-se mostrar, de forma aplicada, que quanto maiores forem essas barreiras, menor será a possibilidade de fazer com que os conhecimentos fluam, e se multipliquem naturalmente. Este estudo originou, por consequiência, uma contribuição ao nível da concepção de diagnóstico organizacional. A perspectiva da organização, através das barreiras cognitivas proposta, apresentase como uma alternativa para a melhor compreensão das capacidades estratégicas, buscando agregar valor na análise para as complexas decisões gerenciais relativas à promoção de ativos intangíveis.

\section{Referências}

ALAVI, M. Review: Knowledge Management and Knowledge Management Systems: Conceptual Foundations and Research Issues. MIS Quarterly, v. 5, n. 1, p. 107-136, março/2001.

BARNEY, J. B. Firm Resources and Sustained Competitive Advantage. Journal of Management. v. 17, p. 99120, 1991.

EDEN, C. \& ACKERMANN, F. The Journal of Strategic Management. London: Sage Publications, 1998.

EISENHARDT, K. M. Building Theories from Case Study Research. Academy of Management Review, v. 14, n. 4, p. 532-550, 1989.

GLADSTEIN, D.; QUINN, J. B. Making Decisions and Producing Action: The Two Faces of Strategy. In: PENNINGS, J. M. e Associados (editores). Organizational Strategy and Change. London: Jossey-Bass Inc. Publishers, 1985.

GONÇALO, C.; BURSTEIN, F.; LEZANA, A. A Cognitive Perspective on Organisational Knowledge
Strategy. In: Proceedings of Third European

Conference on Knowledge Management, p. 274-285,

Trinity College, Dublin, Ireland, 24-25 Setembro, 2002.

GONÇALO, C. Modelo das Barreiras Cognitivas para o Uso da Inteligência Organizacional através de Estratégias de Conhecimento. Tese de Doutorado, PPGEP, UFSC, 2004.

GRANT, R.M. The Knowledge-based View of the Firm: Implications for Management Practice. Long Range Planning, v. 30, n. 3, p. 450-454, 1997.

GRATTON, L. LIVING strategy - putting people at the heart of corporate purpose. London: Financial Times Prentice Hall, 2000.

KETCHEN, D. J.; THOMAS, J. B. \& MCDANIEL, R. R. Process, Content and Context: Synergistic Effects on Organizational Performance. Journal of Management, v. 22, p. 231-257, 1996.

LEONARD-BARTON, D. Core Capabilities and core rigidities: a paradox in managing new product development. Strategic Management Journal, Summer Special Issue, v. 13, p. 111-125, 1992.

MAKADOK, R. Toward a synthesis of the resourcebased and dynamic-capability views of rent creation. Strategic Management Journal, v. 22, p. 387-401, 2001.

MARCH, J. G. The Pursuit of Organizational Intelligence. Massachusetts: Blackwell Publishers Inc., 1999.

MATURANA, H. Cognição, Ciência e Vida Cotidiana. Belo Horizonte: Editora UFMG, 2001.

MINTZBERG, H. The Strategy Concept I: Five Ps For Strategy. California Management Review, v. 30, n. 1, p. 11-24, 1987a.

MYERS, M. D. Qualitative Research in Information Systems. Disponível em http://www.qual.auckland.ac.nz/. Acesso em: 26 Jul. 2002.

NONAKA, I.; TAKEUCHI, H. The Knowledge Creating Company. New York: Oxford University Press, 1995.

NONAKA, I.; KROGH, G. V. \& ABEN, M. Making the Most of Your Company's Knowledge: A Strategic Framework. Long Range Planning, v. 34, p. 421-439, 2001.

PETTIGREW, A.; WHIPP, R. Managing the Twin Processes of Competition and Change - The Role of Intangible Assets. In: LORANGE, P.; CHAKRAVARTHY, 
B.; ROOS, J.; VAN de VEN, A. (editores). Implementing Strategic Processes: Change, Learning and Cooperation. Cambridge, Massachusetts: Blackewell Business, 1994.

PÓR, G. The Quest for Collective Intelligence. In: GOZDZ, K. (editor). Community Building - Renewing Spirit; Learning in Business. San Francisco, USA: Sterling \& Stone, 1995.

PRAHALAD, C. K. ; HAMEL, G. O. The Core Competence of the Corporation. Harvard Business Review, v. 68, p. 79-91, May/June, 1990.

QUINN, J. B.;ANDERSON, P; FINKELSTEIN, S. Managing Professional Intellect: Making the Most of the Best. Harvard Business Review, p. 71-80, March-April, 1996.

QUINN, J. B. Strategic Outsourcing: Leveraging Knowledge Capabilities. Sloan Management Review, Cambridge, Summer, 1999.

SPENDER, J. C. Making knowledge the basis of a dynamic theory of the firm. Strategic Management Journal, v. 17, p. 45-62, Winter - Special Issue, 1996.

SVEIBY, K. E. A knowledge-based theory of the firm to guide in strategy formulation. Journal of Intellectual Capital, v. 2, n. 4, p. 344-358, 2001.

TEECE, D. J.; PISANO, G.; SHUEN, A. Dynamic capabilities and strategic management. Strategic Management Journal, v. 18, p. 509-533, 1997.
VENZIN, M.; KROGH, G. von; ROOS, J. Future research into knowledge management. In: KROGH, G. von; ROOS, J.; KLEINE, D. (editores). Knowing in Firms, Understanding, Managing and Measuring Knowledge. London: SAGE Publications, 1998.

WAGNER, R. K; STERNBERG, R. J. Tacit knowledge and intelligence in the everyday world. In: STERNBERG, R. J. e WAGNER, R. W. Practical intelligence. New York: Cambridge University Press, 1986.

WEICK, K. E. Sensemaking in Organizations. London: SAGE Publications, 1995.

WEICK, K. E.; ROBERTS, K. H. Collective mind in organizations: heedful interrelating on flight decks. Administrative Science Quarterly, v. 38, n. 3, p. 357(25), Sept, 1993.

WIIG, K. M. Knowledge Management Foundations: thinking about thinking - how people and organizations create, represent and use knowledge. Arlington, Texas: Schema Press, 1993.

WIT, B. DE ; MEYER, R. Strategy: Process, Content, Context. London: International Thomson Business Press, 1998.

YIN, R. C. Estudo de Caso: planejamento e métodos. Porto Alegre: Bookman, 2001.

ZACK, M. H. Managing codified knowledge. Sloan Management Review, v. 40, i4, p. 45-58, Summer, 1999. 